\title{
Les premières expressions du génome embryonnaire au cours du développement chez différentes espèces animales
}

Le développement précoce d'un embryon est caractérisé par une première période de divisions cellulaires et une deuxième période de mise en place des trois feuillets embryonnaires fondamentaux (ectoderme, mésoderme et endoderme) appelée gastrulation. On s'accorde à penser que la première étape de divisions se déroule conformément à un programme génétique «pré-établi» au cours de l'ovogenèse. Il s'accumule en effet dans l'ovocyte une énorme réserve de molécules (protéines et ARN messagers maternels) qui interviendront au cours des premières divisions au début du développement [1]. La durée de vie des ARNm maternels est très variable d'une molécule à l'autre, et leur utilisation après la fécondation impose leur remplacement total par la mise en activité des gènes exprimés directement à partir du génome de l'embryon. Ce remplacement constitue le passage d'un contrôle maternel à un contrôle zygotique du développement (figure 1). Parmi les transcrits zygotiques synthétisés, $80 \%$ à $90 \%$ correspondent à des transcrits semblables aux transcrits maternels [1]. Le reste de l'activité zygotique (10\% à $20 \%)$ représente une catégorie d'ARN ne faisant pas partie de la réserve maternelle et qui pourraient être transcrits plus ou moins précocement depuis la fécondation.

La transition progressive du contrôle maternel au contrôle zygotique intervient à des moments différents en fonction des espèces: chez l'oursin et le poisson-zèbre (zebra-fish) au dixiè- drosophile au stade nucléaire 14 [3]; chez les amphibiens au onzième cycle cellulaire (axolotl) [4] ou au douzième (xénope) [5]; chez la souris au stade 2 cellules [6]. Cette transition coïncide souvent avec une période considérée comme la période d'activation transcriptionnelle majeure du génome zygotique. Jusqu'à présent, les amphibiens ont servi de modèle pour étudier cette transition. Les particularités de leur développement précoce permettent en effet de faire coïncider cette période d'activation transcriptionnelle majeure du génome avec une période qualifiée de transition blastuléenne, plus connue sous le terme MBT (mid blastula transition).

\section{La transition blastuléenne (MBT)}

Dans un grand nombre d'espèces animales, les premiers clivages de l'œuf sont rapides et synchrones, essentiellement composés de phases $\mathrm{S}$ et M. Intervient ensuite une période de divisions plus lentes et asynchrones. Chez les amphibiens, le passage de la phase des divisions synchrones à la phase de désynchronisation a été initialement défini comme la transition blastuléenne chez l'axolotl [4] ou MBT chez le xénope [7]. On ignore actuellement de quoi dépend le moment de la mise en place de la MBT. Ce moment ne semble pas dépendre d'un mécanisme lié au nombre de divisions cellulaires ou de cycles de replication de l'ADN, ni au temps écoulé depuis la fécondation. C'est durant cette période que s'amorce la mobilité cellulaire et que les transcriptions zygotiques, très discrètes jusqu'alors, deviennent décelables (m/s n०8/9, vol. 10, p. 917) [8]. La nature du ou des facteurs susceptibles d'induire la MBT n'a pas été déterminée à ce jour. Le modèle initial [9] suggère la présence d'un facteur accumulé au cours de l'ovogenèse dans le cytoplasme de l'ovocyte et qui disparaît du cytoplasme après la fécondation pour se localiser sélectivement dans les noyaux et probablement interagir avec l'ADN. Quand ce facteur est épuisé dans le cytoplasme à la suite des nombreuses divisions, les noyaux entrent en phase G1 pour la première fois afin de produire de novo ce facteur qui permet aux cellules de poursuivre leur première phase G1 et d'entrer en phase S du cycle cellulaire. Ce modèle implique donc la titration d'un facteur cytoplasmique d'origine maternelle par du matériel nucléaire. La MBT représente ainsi une étape impliquée dans le passage du contrôle maternel au contrôle zygotique au cours du développement précoce chez l'amphibien.

A partir de quel moment les transcriptions zygotiques deviennent-elles nécessaires pour la poursuite du développement?

Pour préciser le rôle des transcriptions zygotiques avant la MBT, des expériences utilisant des inhibiteurs de transcription comme l' $\alpha$-amaniti- 
ne, inhibiteur de l'ARN polymérase II, ont été réalisées dans de nombreuses espèces [1]. Après un traitement par cette substance, les clivages se déroulent normalement chez l'ascidie, les mollusques, les insectes, les poissons et les amphibiens, mais les embryons ne survivent pas au-delà de la gastrulation précoce. Chez la souris, une à deux divisions peuvent s'effectuer. La réalisation des premières divisions après la fécondation est donc indépendante d'une transcription de novo puisqu'un traitement par l' $\alpha$-amanitine ne modifie pas le déroulement normal de ces divisions. Le blocage du développement suggère cependant la nécessité de transcriptions zygotiques pour la poursuite ultérieure du développement. D'un point de vue méthodologique, il est néanmoins important de préciser que, dans ces expériences, la micro-injection de l' $\alpha$-amanitine s'effectue toujours dès le stade une cellule. L'inhibition agit donc sur toutes les transcriptions zygotiques de l'embryon précoce. L'arrêt du développement pourrait être la conséquence de l'inhibition de la transcription de gènes nécessaires au moment de la gastrulation. Mais on peut aussi envisager que cet arrêt du développement soit la conséquence de l'inhibition de la synthèse de certains transcrits au moment de la MBT ou bien avant la MBT et dont la fonction ne serait pas nécessaire pour la poursuite immédiate du développement mais avec un effet différé, à partir de la gastrulation, par exemple. Cette dernière hypothèse suggérerait alors l'existence de transcrits zygotiques exprimés très précocement au cours des premières divisions de l'œuf pendant le développement normal.

A partir de quel stade les premiers transcrits zygotiques sont-ils détectables au cours du développement?

Différents travaux témoignent de l'existence de transcriptions dans les heures suivant la fécondation chez des organismes aussi divergents que les vers nématodes (ascaris et Caenorhabditis elegans), la sangsue, la droso-

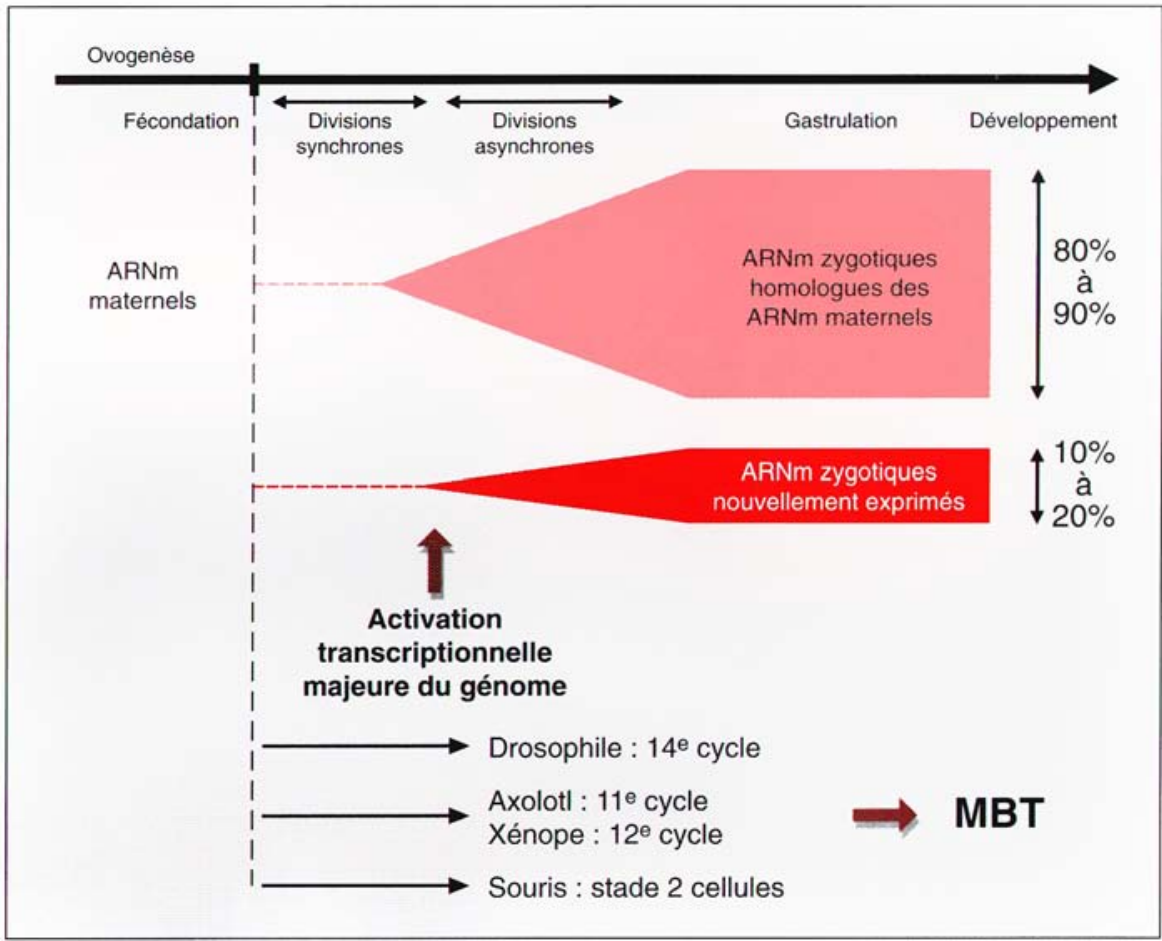

Figure 1. Début du développement embryonnaire. II est schématisé par une flèche depuis l'ovogenèse, la fécondation, l'étape de divisions cellulaires suivie de la gastrulation. L'activité zygotique peut être considérée comme la somme de deux processus : d'une part, le remplacement de transcrits maternels par leurs homologues zygotiques (80 à 90\%) et, d'autre part, l'apparition de nouveaux transcrits, absents de l'héritage maternel et exprimés pour la première fois à partir du génome embryonnaire (10 à $20 \%$ ). L'activation transcriptionnelle majeure du génome intervient à des moments différents en fonction des espèces: quatorzième cycle chez la drosophile, onzième ou douzième cycle de divisions chez les amphibiens axolotl et xénope, stade deux cellules chez la souris. Cette période coïncide avec la MBT (mid blastula transition) initialement définie chez l'axolotl. Une transcription zygotique plus précoce est cependant décrite chez de nombreux organismes depuis la fécondation et pendant les premières divisions synchrones. Elle est représentée par des pointillés rouges sur le schéma.

phile, l'oursin, les amphibiens (axolotl et xénope), la caille et les mammifères (souris) (Tableau I). Cette synthèse d'ARN correspond à divers types de molécules (ARN de transfert, petit ARN nucléaire et ARN ribosomique), mais aussi à des ARN messagers ou à des précurseurs. Elle se produit dès le stade une cellule chez l'oursin ou la souris, à un stade légèrement plus tardif chez l'ascaris ( 4 cellules), Caenorhabditis ( 8 cellules), la sangsue (16 cellules), ou bien au stade 32 noyaux (drosophile), 32 cellules (axolotl et xénope) ou 50 cellules chez la caille (pour revue voir [20]). La transcription de gènes spécifiques a été mise en évidence très précocement chez la sangsue (gène htr-wnt-1) [15], la drosophile (gènes histone et engrailed) [21] et l'ascaris (gène actine) [13], grâce à l'utilisation de sondes moléculaires. Cette transcription zygotique précoce se met donc en route bien avant l'amorce de la transcription majeure considérée comme le début de la transition d'un contrôle maternel à un contrôle zygotique. Il pourrait donc ne pas exister de quiescence transcriptionnelle après la fécondation pour un grand nombre d'organismes en développement. 


\begin{tabular}{|c|c|c|c|}
\hline \multicolumn{4}{|c|}{$\begin{array}{c}\text { Tableau I } \\
\text { PREMIERS TRANSCRITS ZYGOTIQUES }\end{array}$} \\
\hline $\begin{array}{c}\text { Organisme } \\
\text { (espèce) }\end{array}$ & $\begin{array}{l}\text { Moment de l'activation } \\
\text { transcriptionnelle majeure } \\
\text { du génome }\end{array}$ & $\begin{array}{l}\text { Mise en évidence } \\
\text { des premières activités } \\
\text { transcriptionnelles }\end{array}$ & Références \\
\hline $\begin{array}{c}\text { Ascaris } \\
\text { (vers nématode) }\end{array}$ & ND & Stade $4-8$ cellules & [13] \\
\hline $\begin{array}{l}\text { Caenorhabditis } \\
\text { elegans } \\
\text { (vers nématode) }\end{array}$ & Stade $90-125$ cellules & $\begin{array}{c}\text { Entre les stades } 1 \text { et } 30 \\
\text { cellules }\end{array}$ & [14] \\
\hline $\begin{array}{c}\text { Sangsue } \\
\text { (vers annélide) }\end{array}$ & $\begin{array}{c}\text { Stade } 7 \\
\text { (100-1000 cellules) }\end{array}$ & $\begin{array}{c}\text { Stade } 4-5 \\
\text { ( } 25 \text { cellules })\end{array}$ & [15] \\
\hline $\begin{array}{l}\text { Drosophile } \\
\text { (insecte) }\end{array}$ & Cycle 14 & Cycle 6-9 & {$[16,21]$} \\
\hline $\begin{array}{c}\text { Oursin } \\
\text { (échinoderme) }\end{array}$ & $\begin{array}{l}\text { Pas de quiescence } \\
\text { transcriptionnelle } \\
\text { après fécondation }\end{array}$ & A partir du cycle 1 & [10] \\
\hline $\begin{array}{c}\text { Caille } \\
\text { (oiseaux) }\end{array}$ & ND & $\begin{array}{l}\text { Au cours des premières } \\
\text { divisions cellulaires }\end{array}$ & [19] \\
\hline $\begin{array}{c}\text { Axolotl } \\
\text { (amphibien urodèle) }\end{array}$ & $\begin{array}{c}\text { Cycle } 11 \\
\text { (environ } 500 \text { cellules) }\end{array}$ & $\begin{array}{c}\text { Depuis le cycle } 1 \\
\text { et au stade } 32 \text { cellules }\end{array}$ & [17] \\
\hline $\begin{array}{c}\text { Xénope } \\
\text { (amphibien anoure) }\end{array}$ & $\begin{array}{c}\text { Cycle } 12 \\
\text { (environ } 2000 \text { cellules) }\end{array}$ & Stade 32 cellules & {$[7,8,18]$} \\
\hline $\begin{array}{c}\text { Souris } \\
\text { (mammifère) }\end{array}$ & Stade 2 cellules & Stade 1 cellule & {$[11,12]$} \\
\hline
\end{tabular}

$N D$ : non déterminé.

La dégradation des ARNm maternels et l'activation de la transcription zygotique sont-ils des processus interdépendants?

Dans la plupart des espèces, la transition du contrôle maternel au contrôle zygotique coincide avec deux processus: la dégradation des ARNm maternels et l'activation de la transcription zygotique. Quelle est l'influence du premier phénomène sur le second? Chez la drosophile, l'étude du gène String a apporté une réponse (figure 2). String est l'homologue du gène $c d c 25$ de Schizosaccharomyces pombe, nécessaire à la déphosphorylation de la kinase p34 $4^{c d c 2}$ [22]. transcription de string subit une modification importante [23, 24]. Pendant les treize premiers cycles embryonnaires, les ARNm maternels string sont stables. Ils sont totalement dégradés au tout début de la phase $\mathrm{S}$ du cycle 14. La transcription zygotique de string ne débute qu'à partir de la phase G2 du cycle 14. Lorsque l' $\alpha$-amanitine est micro-injectée dans des embryons au cours du cycle 14, après la dégradation des ARNm maternels string, mais avant l'expression zygotique, la mitose qui suit est bloquée. Un phénotype identique est observé avec les mutants string dont le développement est arrêté avant la mitose 14. La similitude de ces deux phénotypes suggère qu'une trans- cription zygotique est nécessaire en phase G2 du cycle 14 . Lorsque l' $\alpha$ amanitine est injectée plus précocement (cycle 9), le phénotype d'arrêt du développement est différent des phénotypes précédents. L'embryon continue son cycle 14 et le blocage se produit après la quatorzième mitose. La réalisation de cette mitose peut être interprétée comme la conséquence d'une persistance inhabituelle des transcrits maternels string pendant le cycle 14. Ces ARNm maternels ne seraient apparemment pas dégradés au cycle 13, comme dans l'embryon normal, et permettraient la réalisation de la mitose 14 . La présence d' $\alpha$-amanitine à partir du cycle 9 pourrait alors inhiber 


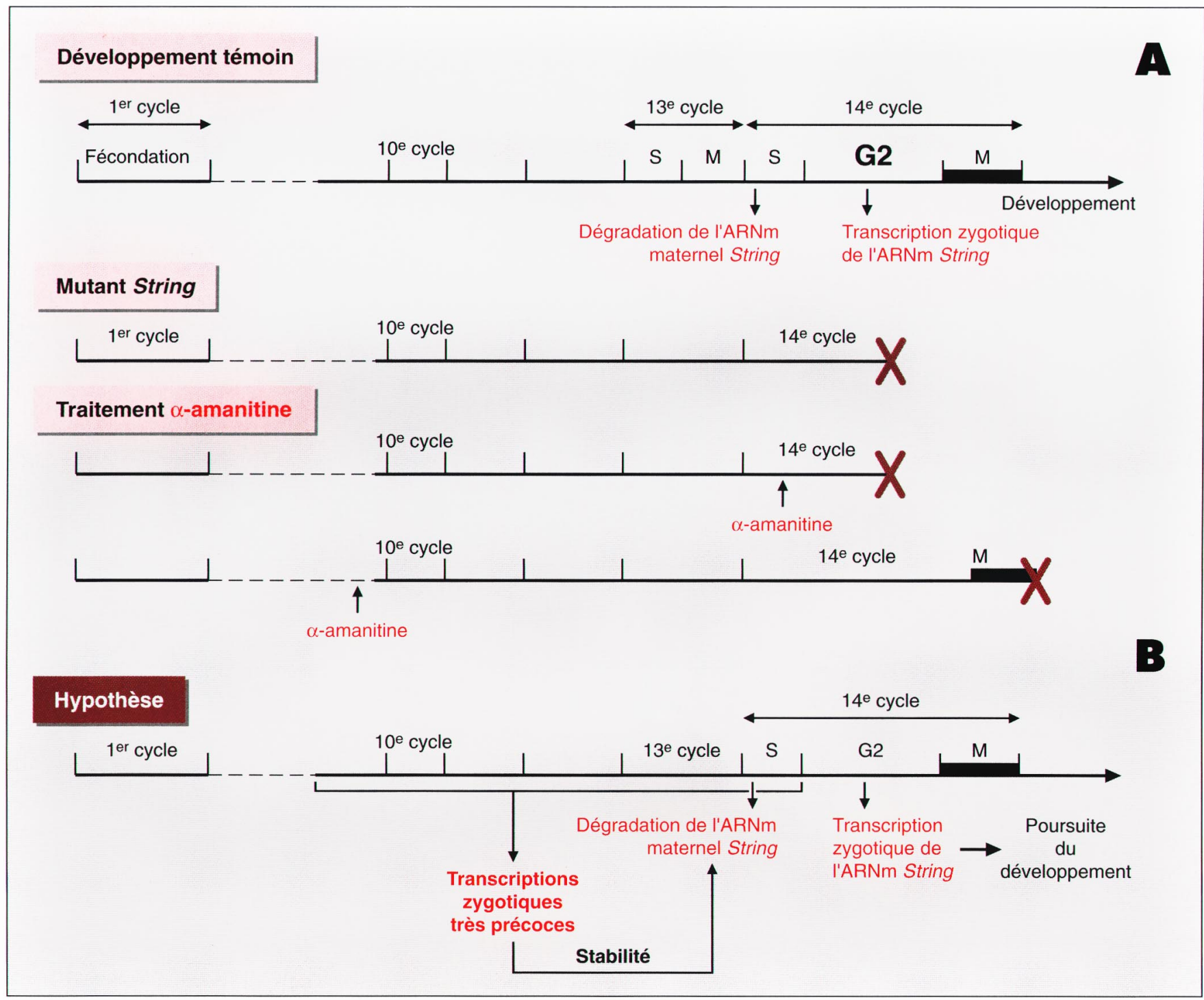

Figure 2. A. Le développement embryonnaire chez la drosophile est constitué de 13 premiers cycles composés de phases $S$ et M. Une phase G2 s'instaure au cycle 14. La dégradation totale des ARNm maternels string s'effectue au début de la phase $S$ du cycle 14. La transcription zygotique de string ne débute qu'au cours de la phase G2 du cycle 14. Lorsque I' $\alpha$-amanitine est injectée pendant la phase $S$ du cycle 14, après la dégradation des ARNm maternels string, un arrêt du développement se produit en G2 du cycle 14 comme dans les embryons mutants strings. Lorsque I' $\alpha$-amanitine est injectée avant le cycle 10, l'embryon continue son cycle 14 et le blocage intervient après la mitose 14. B. Modèle hypothétique impliquant l'existence de transcriptions zygotiques très précoces dans la stabilité d'ARNm maternels. Ces transcriptions zygotiques interviendraient dans la dégradation spécifique d'ARNm maternels dont I'ARNm string de façon à permettre l'activation transcriptionnelle majeure zygotique nécessaire à la poursuite du développement.

l'expression de molécules zygotiques précoces impliquées dans la régulation spécifique des ARNm maternels dont l'ARNm string [25]. L'ensemble de ces données suggèrent la nécessité d'une expression zygotique très précoce dont l'un des rôles serait de permettre le passage du contrôle mater- nel au contrôle zygotique en dégradant certains ARNm maternels spécifiques. Sans exclure la possibilité que d'autres mécanismes puissent être impliqués dans l'activation transcriptionnelle majeure du génome (synthèse de novo de facteurs de transcription et/ou modulation de la demi-vie des ARNm), cette transition pourrait résulter d'au moins trois processus non exclusifs: (1) une modification du rapport nucléo-cytoplasmique (titration d'un facteur cytoplasmique maternel); (2) une réduction de la stabilité des ARN par une dégradation spécifique de transcrits mater- 
nels; (3) une activation zygotique très précoce de gènes impliqués dans le contrôle de cette dégradation.

L'isolement et la caractérisation de ces transcrits très précoces pourraient permettre de mieux comprendre leur rôle dans la régulation temporelle des premiers clivages embryonnaires. On peut supposer qu'ils pourraient intervenir dans la déstabilisation spécifique de certains ARNm maternels de façon à permettre l'activation majeure de l'expression des gènes zygotiques nécessaire au développement de l'embryon

\section{RÉFÉRENCES}

1. Davidson EH. Gene activity in early development, $3^{\text {rd }}$ ed. New York: Academic Press, 1986.

2. Kane DA, Kimmel CB. The zebrafish midblastula transition. Development 1993; 119: 447-56.

3. Yasuda GK, Schubiger G. Temporal regulation in the early embryo: is MBT too good to be true? Trends Genet Sci 1992; 8 : 124-7.

4. Signoret J, Lefresne J. Contribution à l'étude de la segmentation de l'œuf d'axolotl. I - Définition de la transition blastuléenne. Ann Embr Morph 1971; 4: 113-23.

5. Newport J, Kirschner M. A major development transition in early Xenopus embryos : I-Characterization and timing of cellular changes at the midblastula stage. Cell 1982; 30:675-86.

6. Schultz RM. Regulation of zygotic gene activation in the mouse. Bioessays $1993 ; 15$ : 531-8.

7. Newport J, Kirschner M. A major developmental transition in early Xenopus embryos. II - Control of the onset of transcription. Cell 1982; $30: 687-96$.
8. Prioleau MN, Huet J, Sentenac A, Méchali M. Competition between chromatin and transcription complex assembly regulates gene expression during early development. Cell 1994; 77; 439-49.

9. Signoret J, Lefresne J. Contribution à l'étude de la segmentation de l'œuf d'axolotl. II - Influence de modifications du noyau et du cytoplasme sur les modalités de la segmentation. Ann Embr Morph 1973; 6 : 299-307.

10. Poccia D, Wolff R, Kragh S, Williamson P. RNA synthesis in male pronuclei of the sea urchin. Biochem Biophys Acta 1985; 824 : 349-56.

11. Clegg KB, Piko L. RNA synthesis and cytoplasmic polyadenylation in the one-cell mouse embryo. Nature 1982; 295 : 342-5.

12. Christians E, Campion E, Thompson EM, Renard JP. Expression of the HSP 70.1 gene, a landmark of early zygotic activity in the mouse embryo, is restricted to the first burst of transcription. Development 1995; 121: 113-22.

13. Cleavinger PJ, McDowell JW, Bennett KL. Transcription in nematodes: early ascaris embryos are transcriptionally active. Dev Biol 1989; 133 : 600-4.

14. Schauer IE, Wood WB. Early C. elegans embryos are transcriptionally active. Development 1990 ; 110 : 1303-17.

15. Bissen ST, Weisblat DA. Transcription in leech: mRNA synthesis is required for early cleavage in Helobdella embryos. Dev Biol $1991 ; 146$ : 12-23.

16. Gutzeit HO. Expression of the zygotic genome in blastoderm stage embryos of Drosophila - Analysis of a specific protein. Wilhelm Roux's Archives 1980; 188 : 153-6.

17. Signoret J, Lefresne J. Mise en évidence d'une RNA synthase en cours de segmentation chez le germe d'axolotl. CR Acad Sci Paris 1975 ; 279 : 1189-91.

18. Kimelman D, Kirschner M, Scherson T. The events of the midblastula transition in Xenopus are regulated by changes in the cell cycle. Cell 1987; 48 : 399-407.

19. Olszanska B, Kludkiewicz B, Lassota Z. Transcription and polyadenylation processes during early development of quail embryo. $J$ Embryol Exp Morph 1984; 79: 11-24.
20. Andéol Y. Early transcription in different animal species: implication for transition from maternal to zygotic control in development. Roux's Arch Dev Biol 1994; 204 : 3-10.

21. Karr TL, Weir MP, Ali Z, Kornberg T. Patterns of engrailed proteins in early Drosophila embryos. Development 1989; 105 : 60512.

22. Edgar BA, O'Farrell PH. The three postblastoderm cell cycles of Drosophila embryogenesis are regulated in G2 by string. Cell $1990 ; 62$ : 469-80.

23. O'Farrell PH, Edgar BA, Lakich D, Lehner CF. Directing cell division during development. Science 1989; 246 : 635-40.

24. Edgar BA, O'Farrell PH. Genetic control of cell division patterns in the Drosophila embryo. Cell 1989; 57 : 177-87.

25. Yasuda GK, Baker J, Schubiger G. Temporal regulation of gene expression in the blastoderm Drosophila embryo. Cell 1991; 5 : 1800-12.

\section{Remerciements}

L'auteur remercie Luisa Dandolo et Joël Aghion pour les critiques constructives apportées à la rédaction de ce travail.

\section{Yannick Andéol}

Maître de conférences à l'université ParisVI. Laboratoire de biologie cellulaire et moléculaire, unité de biologie du développement, Inra, 78350 Jouy-en-Josas, France.

\section{TIRÉS À PART}

Y. Andéol. 